Research Paper

\title{
Integrin $\beta 3$ promotes cardiomyocyte proliferation and attenuates hypoxia-induced apoptosis via regulating the PTEN/Akt/mTOR and ERK1/2 pathways
}

\author{
Lijiang Wei' ${ }^{1}$ Qingqing Zhou ${ }^{2}$, Hua Tian ${ }^{2 凶}$, Yifan Su${ }^{1}$, Guo-hui Fu ${ }^{3}$, Ting Sun ${ }^{1 凶}$ \\ 1. Department of Cardiology, Shanghai Ninth People's Hospital, Shanghai Jiaotong University School of Medicine. Shanghai, 200025, China \\ 2. State Key Laboratory of Oncogenes and Related Genes, Shanghai Cancer Institute, Renji Hospital, Shanghai Jiaotong University School of Medicine, \\ Shanghai, 20032, China \\ 3. Pathology Center, Shanghai General Hospital/Faculty of Basic Medicine, Shanghai Jiaotong University School of Medicine, No.280, South Chong-Qing \\ Road, Shanghai 200025, People's Republic of China
}

$\triangle$ Corresponding authors: Ting Sun, MD, Department of Cardiology, Shanghai Ninth People's Hospital Affiliated with Shanghai Jiaotong University School of Medicine, Shanghai 200025, China. Tel/Fax: +86-86-21-23271287; E-mail: beibeisun2008@163.com. Hua Tian, MD, State Key Laboratory of Oncogenes and Related Genes, Shanghai Cancer Institute, Renji Hospital, Shanghai Jiaotong University School of Medicine, 25/Ln 2200, Xietu Road, Shanghai 200032, China. Tel/Fax: +86-21-64432140; E-mail: htian@shsci.org

(C) The author(s). This is an open access article distributed under the terms of the Creative Commons Attribution License (https://creativecommons.org/licenses/by/4.0/). See http://ivyspring.com/terms for full terms and conditions.

Received: 2019.08.20; Accepted: 2019.12.02; Published: 2020.01.14

\begin{abstract}
Objective: Integrin $\beta 3$ is one of the main integrin heterodimer receptors on the surface of cardiac myocytes. Our previous studies showed that hypoxia induces apoptosis and increases integrin $\beta 3$ expression in cardiomyocytes. However, the exact mechanism by which integrin $\beta 3$ protects against apoptosis remains unclear. Hence, the present investigation aimed to explore the mechanism of integrin $\beta 3$ in cardiomyocyte proliferation and hypoxia-induced cardiomyocyte apoptosis.

Methods: Stable cells and in vivo acute and chronic heart failure rat models were generated to reveal the essential role of integrin $\beta 3$ in cardiomyocyte proliferation and apoptosis. Western blotting and immunohistochemistry were employed to detect the expression of integrin $\beta 3$ in the stable cells and rat cardiac tissue. Flow cytometer was used to investigate the role of integrin $\beta 3$ in hypoxia-induced cardiomyocyte apoptosis. Confocal microscopy was used to detect the localization of integrin $\beta 3$ and integrin $\alpha v$ in cardiomyocytes.

Results: A cobaltous chloride-induced hypoxic microenvironment stimulated cardiomyocyte apoptosis and increased integrin $\beta 3$ expression in $\mathrm{H} 9 \mathrm{C} 2$ cells, $\mathrm{ACl} 6$ cells, and cardiac tissue from acute and chronic heart failure rats. The overexpression of integrin $\beta 3$ promoted cardiomyocyte proliferation, whereas silencing integrin $\beta 3$ expression resulted in decreased cell proliferation in vitro. Furthermore, knocking down integrin $\beta 3$ expression using shRNA or the integrin $\beta 3$ inhibitor cilengitide exacerbated cobaltous chloride-induced cardiomyocyte apoptosis, whereas overexpression of integrin $\beta 3$ weakened cobaltous chloride-induced cardiomyocytes apoptosis. We found that integrin $\beta 3$ promoted cardiomyocytes proliferation through the regulation of the PTEN/Akt/mTOR and ERK1/2 signaling pathways. In addition, we found that knockdown of integrin $\alpha v$ or integrin $\beta 1$ weakened the effect of integrin $\beta 3$ in cardiomyocyte proliferation.

Conclusion: Our findings revealed the molecular mechanism of the role of integrin $\beta 3$ in cardiomyocyte proliferation and hypoxia-induced cardiomyocyte apoptosis, providing new insights into the mechanisms underlying myocardial protection.
\end{abstract}

Key words: integrin $\beta 3$; PTEN/Akt/mTOR; apoptosis; cardiomyocytes; hypoxia

\section{Introduction}

Heart failure is a major public health concern because of its high mortality, high rate of hospitalization, and cost of management. Heart failure can be categorized into acute heart failure and 
chronic heart failure. Heart failure leads to hypoxia in cells and tissues. In turn, hypoxia exacerbates the deterioration of heart failure patients, thereby establishing a vicious circle of increasing hypoxia and subsequent malignant progression [1]. Therefore, the identification of new diagnostic and therapeutic targets in hypoxia-induced heart failure for improving the prognosis of heart failure patients is required.

Integrins are widely expressed on the cell surface and involve cell-extracellular matrix adhesion and interaction. The integrin family is composed of $24 \alpha \beta$ heterodimeric members. Integrin heterodimers consist of one $\alpha$ and one $\beta$ subunit [2]. Accumulating evidence has shown that the expression of integrins is associated with cell proliferation, migration, invasion, differentiation, and matrix remodeling [3]. Integrin $\beta 3$ is one of the main integrin heterodimer receptors on the surface of cardiomyocytes [4]. Extensive studies have shown that integrin $\beta 3$ contributes to the survival, proliferation and metastatic phenotype of human tumors $[2,5]$. Previous studies have shown that integrin $\beta 3$ knockout mice exhibit myocardial cell apoptosis in pressure-overload hypertrophy [6]. The overexpression of integrin $\beta 3$ inhibites lipopolysaccharide (LPS)-induced autophagy in cardiomyocytes [7]. Recent studies have shown that integrin $\beta 3$ is required for the attachment, retention and therapeutic benefits of human cardiospheres in myocardial infarction [8]. Therefore, these results suggest that integrin $\beta 3$ plays an important role in cardiovascular disease. Our previous studies showed that integrin $\beta 3$ inhibits hypoxia induced apoptosis in cardiomyocytes [9]. However, the exact mechanism by which integrin $\beta 3$ protects against apoptosis remains unclear. In this study, we investigated the exact mechanism of the role of integrin $\beta 3$ in cardiomyocyte proliferation and hypoxia-induced cardiomyocyte apoptosis.

\section{Materials and Methods}

\section{Cell lines and cell culture}

The rat embryonic cardiomyocyte cell line H9C2 was purchased from the cell bank of the Institute of Biochemistry and Cell Biology of the Chinese Academy of Sciences (Shanghai, China). The human cardiac cell line AC16 was purchased from American Type Culture Collection (ATCC, Rockville, MD). These cell lines were continuously cultured in Dulbecco's modified Eagle's medium (DMEM) containing $10 \%$ fetal bovine serum (FBS) at $37^{\circ} \mathrm{C}$ and $5 \% \mathrm{CO}_{2}$.

\section{Primary culture of myocardial cells}

Primary rat myocardial cells were isolated according to a previously described protocol [9]. Primary rat myocardial cells were isolated from the hearts of 1- to 3-day-old Sprague-Dawley rats with $0.25 \%$ trypsin. The cells $\left(1 \times 10^{5}\right.$ cells/well $)$ were continuously cultured in DMEM containing 10\% fetal bovine serum (FBS) at $37^{\circ} \mathrm{C}$ and $5 \% \mathrm{CO}_{2}$.

\section{Plasmids and short hairpin RNAs (shRNAs)}

Vectors expressing integrin $\beta 3$ and control were obtained from Funeng (Guangzhou, China). Short hairpin RNAs (shRNAs) targeting integrin $\beta 3$ and integrin $\beta 1$ and a general negative control (NC) shRNA were synthesized by Funeng (Guangzhou, China). shRNA for integrin av was purchased from GeneChem (Shanghai, China). The fragments were designed to target integrin $\beta 3$, integrin $\beta 1$ and integrin av transcripts. The target sequence of integrin $\beta 3$ was 5'-GCAAACAACCCATTGTATA-3'. The target sequence of integrin $\beta 1-1$ was $5^{\prime}$-CCAGAAGACA TTACTCAGATC-3'. The target sequence of integrin $\beta 1-2$ was $5^{\prime}$-CCATACATTAGTACAACACCA-3'. The target sequence of integrin av was 5'-GATAAGAGGAGTCTCGAGT-3'.

\section{Lentivirus production and cell transduction}

A lentiviral vector (integrin $\beta 3$ ), shRNA vectors (shintegrin $\beta 3$, shintegrin $\beta 1$ and shintegrin av) and packaging vector psPAX2 and pMD2G were cotransfected into HEK293T cells using Lipofectamine 2000 . The supernatant containing the integrin $\beta 3$-overexpressing and integrin $\beta 3$, integrin $\beta 1$ and integrin av knockdown lentiviruses was then harvested after 72 hours and filtered through a $0.45-\mu \mathrm{m}$ sterile syringe. H9C2 and AC16 cells were infected with $1 \times 10^{6}$ recombinant lentivirustransducing units in the presence of $6 \mu \mathrm{g} / \mathrm{ml}$ polybrene (Sigma).

\section{Western blotting}

Western blotting was performed according to a previously described protocol [10]. The cell pellet was washed with cold PBS once and then lysed with RIPA buffer (Thermo Scientific) containing protease inhibitor, and phosSTOP phosphatase inhibitor cocktail (Roche, Welwyn Garden, Swiss, UK). The lysates were centrifuged at 14,000 rotations per minute for 15 minutes at $4^{\circ} \mathrm{C}$, and then the cleared supernatant was collected. The proteins were separated by sodium dodecylsulfate-polyacrylamide gel electrophoresis (SDS-PAGE), electroblotted onto nitrocellulose membranes and probed with primary antibodies. The primary antibodies used for western blotting were against integrin $\beta 3$ (Abcam, Cambridge, 
UK), integrin $\beta 1$ (Abcam, Cambridge, UK), integrin av (Abcam, Cambridge, UK), phosphor-mTOR (Abcam, Cambridge, UK), mTOR (Abcam, Cambridge, UK), phosphor-ERK1/2 (Cell Signaling Technology, USA), ERK1/2 (Cell Signaling Technology, USA), phosphor-Akt (Cell Signaling Technology, USA), Akt (Cell Signaling Technology, USA), Bcl-2 (Proteintech, China), Bax (Proteintech, China), cleaved caspase 3 (Cell Signaling Technology, USA), PTEN (Cell Signaling Technology, USA) and $\beta$-actin (Sigma, USA). The immunoreactive bands were visualized using an ECL reagent (Pierce, Rockford, IL, USA).

\section{Quantitative RT-PCR (qRT-PCR)}

According to the manufacturer's protocol, total RNA was extracted using TRIzol reagent (Invitrogen, Carlsbad, CA, USA), and cDNA was generated from 1 $\mu g$ of total RNA using a Prime-Script RT Reagent Kit (TaKaRa, Shanghai, China). qRT-PCR was performed using a 7500 system (Thermo Scientific, MA, USA).The qRT-PCR primer sequences were as follows: integrin $\beta 3$-F: $5^{\prime}$ - AGTCAGCGAGGCCCAGA TC-3', integrin $\beta 3-R: 5^{\prime}$ - AGGGTCTGGATGCTGGA CAG-3'; $\beta$-actin-F: $5^{\prime}$ - AGGCATCCTGACCCTGAAG TAC-3', and $\beta$-actin-R: $5^{\prime}$ - GAGGCATACAGGGACA ACACAG-3'.

\section{Cell proliferation and colony formation assays}

Cell proliferation was detected using a Cell Counting Kit-8 according to the manufacturer's specifications. Colony formation was evaluated by seeding cells at concentrations of $5 \times 10^{3} /$ well in 6-well plates. Once colonies were visible, they were fixed with formaldehyde solution. Then, the cells were stained with Giemsa. The number of colonies per well was counted. Each experiment was performed in triplicate.

\section{Apoptosis Assay}

An Annexin V-AbFluor ${ }^{\mathrm{TM}} 555$ Apoptosis Detection Kit (Abbkine) was used to visualize apoptotic cells according to the manufacturer's instructions. Briefly, $2 \times 10^{5}$ cells were collected, washed with ice-cold PBS twice and resuspended in $100 \mu \mathrm{L}$ of Annexin V binding buffer. Next, $4 \mu \mathrm{L}$ of Annexin V- AbFlourTM 555 was added to the cell suspension and incubated at room temperature for 15 minutes. Next, $400 \mu \mathrm{L}$ of Annexin V Binding Buffer was added, and the samples were analyzed with a flow cytometer.

\section{Animal and Treatment}

Twenty male Sprague-Dawley rats, aged 8-weeks-old and weighing 220-260 g, were supplied by Jiesijie Laboratory Animal Co. (Shanghai, China) and fed regular food in separate cages at $20-25^{\circ} \mathrm{C}$ and $60-70 \%$ in a humidity-controlled environment. The rats had free access to water that was disinfected with ultraviolet radiation.

Coronary artery ligation was performed as previously described to establish a rat acute myocardial infarction (AMI) model [9]. The same procedure without coronary artery ligation was performed in sham controls. All rats were anesthetized and euthanized $6 \mathrm{~h}$ after coronary artery ligation.

Chronic heart failure (CHF) model rats were intraperitoneally administered isoproterenol (ISO) (10 $\mathrm{mg} / \mathrm{kg}$ ) or an equal volume of saline daily for two weeks to induce heart failure as previously described. After one month, all rats were sacrificed and euthanized. The hearts were removed, cleared of blood, and immediately transferred to ice-cold containers containing $0.9 \%$ sodium chloride.

\section{Immunohistochemistry (IHC)}

Immunohistochemical staining was performed as described previously [9]. Paraffin-embedded tissues were sectioned, blocked with $3 \% \mathrm{H}_{2} \mathrm{O}_{2}$ in PBS for $15 \mathrm{~min}$ at room temperature and then incubated with the indicated antibodies overnight at $4{ }^{\circ} \mathrm{C}$. A horseradish peroxidase (HRP)-conjugated secondary antibody was used, and a DAB kit was employed for signal detection. The control samples were incubated without primary antibody or with normal serum instead of primary antibody.

\section{Statistical analyses}

SPSS 16.0 statistical package (SPSS, Inc., Chicago, IL, USA) or GraphPad Software was used to analyze the data. All results are presented as the mean \pm S.D. Two-group comparisons were analyzed using the two-tailed Student's $t$ test. Comparisons of three or more groups were analyzed using one-way ANOVA. Statistical significance was defined as ${ }^{*} P<0.05$ and $* * P<0.01$.

\section{Results}

The expression of integrin $\beta 3$ and integrin $\alpha v$ is upregulated in $\mathrm{CoCl}_{2}$-induced cardiomyocyte apoptosis

We first detected the expression of integrin $\beta 3$ in $\mathrm{CoCl}_{2}$-induced cardiomyocyte apoptosis. Our results showed that $\mathrm{CoCl}_{2}$ inhibited cardiomyocyte proliferation and induced cardiomyocyte apoptosis in H9C2, AC16 and primary rat myocardial cells (Figure $1 \mathrm{~A}$, Figure S1). We found that the expression of integrin $\beta 3$ was upregulated in $\mathrm{CoCl}_{2}$-induced cardiomyocyte apoptosis (Figure 1B). These results are consistent with our previous finding [9]. To 
confirm the role of integrin $\beta 3$ in hypoxia-induced cardiomyocyte apoptosis, the expression of integrin $\beta 3$ was also detected in AMI and CHF rat cardiac tissue. Our IHC results also showed that the expression of integrin $\beta 3$ was upregulated in AMI and $\mathrm{CHF}$ rat cardiac tissue (Figure $1 \mathrm{C}-1 \mathrm{~F}$ ).

Integrin av and $\beta 3$ subunits noncovalently bind to form an integrin av $\beta 3$ heterodimer [12]. Therefore, we also detected the expression of integrin av in hypoxia-induced cardiomyocyte apoptosis. Our results showed that the expression of integrin av was upregulated in hypoxia-induced cardiomyocytes in vitro and in vivo (Figure 1B, Figure 1C-1F). These results showed that integrin av $\beta 3$ can play an important role in cardiomyocyte apoptosis.

\section{Integrin $\beta 3$ promotes cardiomyocyte proliferation and inhibits $\mathrm{CoCl}_{2}$-induced cardiomyocyte apoptosis}

We next explored the role of integrin $\beta 3$ in cardiomyocyte proliferation and apoptosis. Using a lentiviral vector system, we successfully established
H9C2 and AC16 cell lines with stable ectopic integrin $\beta 3$ expression and stable integrin $\beta 3$ knockdown. The efficiency of the overexpression and knockdown of integrin $\beta 3$ was verified by qRT-PCR and western blotting (Figure 2A-2D). Our results showed that the overexpression of integrin $\beta 3$ increased cardiomyocyte proliferation and clone-forming ability (Figure 2E and 2F). Conversely, the knockdown of integrin $\beta 3$ inhibited cardiomyocyte proliferation and clone-forming ability (Figure $2 \mathrm{G}$ and $2 \mathrm{H}$ ). Therefore, these results suggested that integrin $\beta 3$ promotes cardiomyocyte proliferation.

We next detected the expression of apoptosis-related proteins during the promotion of cardiomyocyte proliferation by integrin $\beta 3$. Our results showed that cleaved caspase 3 and pro-apoptotic protein Bax was decreased and that the anti-apoptotic protein $\mathrm{Bcl}-2$ was increased in integrin $\beta 3$-overexpressing $\mathrm{H} 9 \mathrm{C} 2$ and AC16 cells (Figure 3A, Figure S2A). The cleaved caspase 3 and pro-apoptotic protein Bax was increased, and the anti-apoptotic protein Bcl-2 was decreased in integrin $\beta 3$ knockdown
A
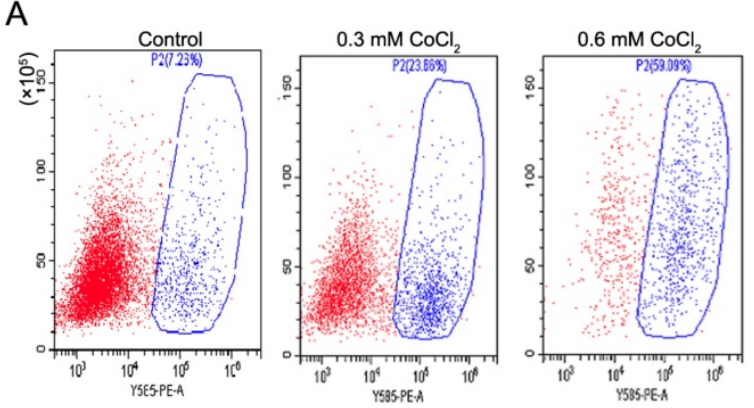

C

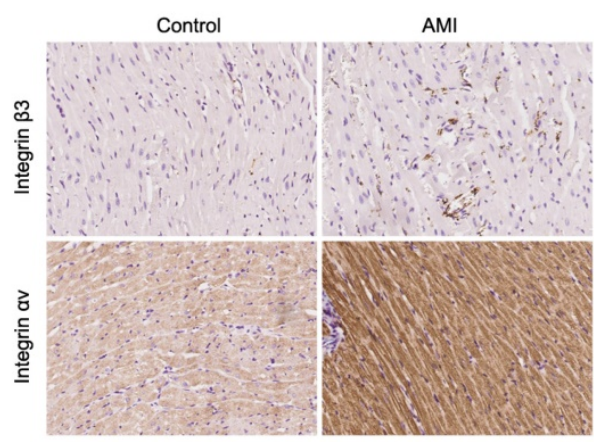

E
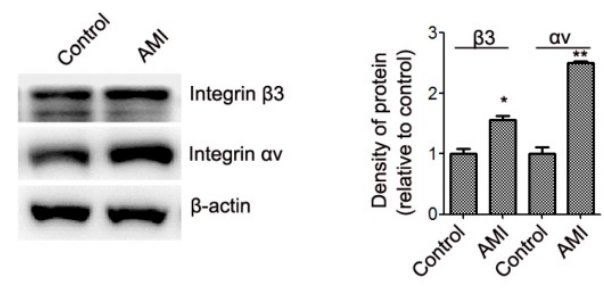
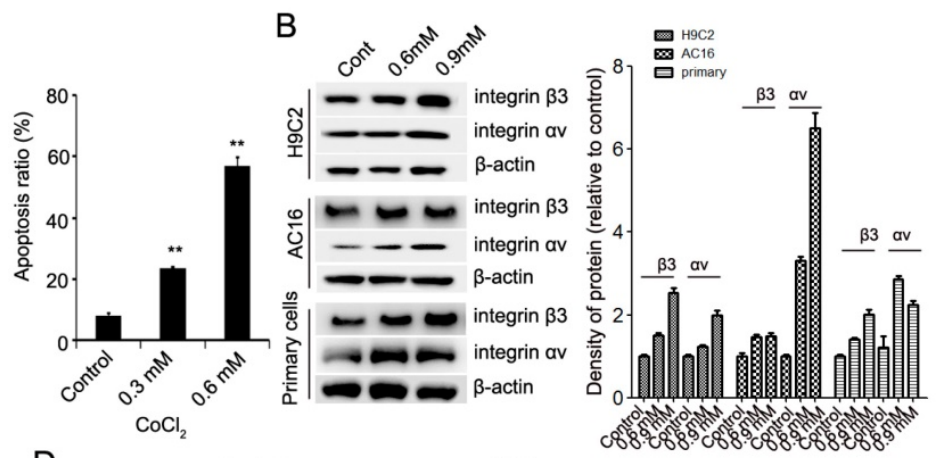

D

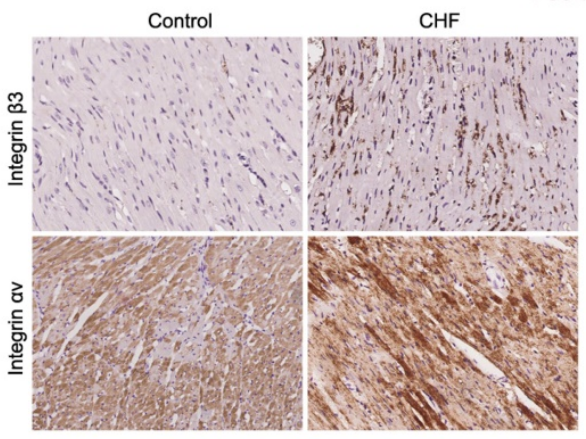

F
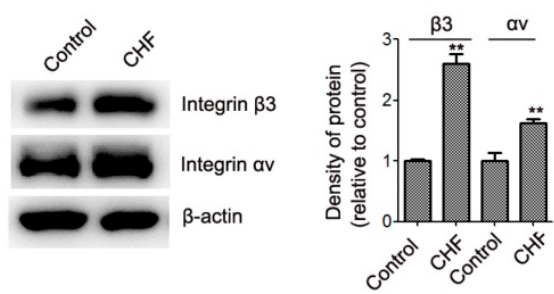

Figure 1. Expression of integrin $\beta 3$ and integrin $\alpha v$ in hypoxia-treated cardiomyocytes and myocardial tissue from heart failure rats. (A) H9C2 cells were treated with $\mathrm{CoCl}_{2}(600 \mu \mathrm{M})$ for 12 hours. Cell apoptosis was detected by flow cytometry. (B) Integrin $\beta 3$ and integrin av expression was detected by western blot analysis in $\mathrm{H} 9 \mathrm{C} 2, \mathrm{ACl} 16$ and primary rat myocardial cells treated with $\mathrm{CoCl}_{2}{ }^{*} \mathrm{P}<0.05 ; * * P<0.01$. (C) Immunohistochemical analysis of integrin $\beta 3$ and integrin $\alpha \mathrm{v}$ in the control and $\mathrm{AMI}$ groups. (D) Immunohistochemical analysis of integrin $\beta 3$ and integrin $\alpha v$ in the control and CHF groups. Representative images are shown $(\times 200)$. (E) Western blot analysis of integrin $\beta 3$ and integrin $\alpha v$ in the control and AMI groups. (F) Western blotting analysis of integrin $\beta 3$ and integrin $\alpha v$ in the control and CHF groups. 
H9C2 and AC16 cells (Figure 3B, Figure S2B). Therefore, these results suggested that integrin $\beta 3$ promotes cardiomyocyte proliferation and inhibits cardiomyocyte apoptosis.

We also examined the effect of integrin $\beta 3$ on $\mathrm{CoCl}_{2}$-induced cardiomyocyte apoptosis. The results showed that the overexpression of integrin $\beta 3$ inhibited $\mathrm{CoCl}_{2}$-induced cardiomyocyte apoptosis, whereas the knockdown of integrin $\beta 3$ increased $\mathrm{CoCl}_{2}$-induced cardiomyocyte apoptosis (Figure 2I).

Cilengitide is an integrin $\operatorname{av} \beta 3$ receptor antagonist. We detected whether cilengitide exacerbates $\mathrm{CoCl}_{2}$-induced cardiomyocyte apoptosis. Our results showed that cilengitide inhibited the expression of integrin av and $\beta 3$ in $\mathrm{CoCl}_{2}$-treated cells (Figure 4A). Furthermore, cilengitide exacerbated apoptosis and the inhibition of proliferation in $\mathrm{CoCl}_{2}$-induced cardiomyocytes (Figure 2I, Figure 4B).
In addition, we found that cilengitide inhibited H9C2 and AC16 cells proliferation in a dose-dependent manner. The expression of cleaved caspase 3 was upregulated in the cilengitide-treated cells (Figure 4C and 4D). Therefore, these results showed that integrin $\beta 3$ promotes cardiomyocyte proliferation and inhibits hypoxia-induced cardiomyocyte apoptosis.

\section{$\mathrm{CoCl}_{2}$ inhibits cardiomyocyte proliferation through the regulation of the PTEN/Akt/mTOR and ERK1/2 signaling pathways}

Recent evidence has implicated mTOR as a central regulator of proliferation in various normal and malignant cells [13, 14]. Therefore, we detected the expression of mTOR inCoCl $\mathrm{Cl}_{2}$-treated cardiomyocytes. Our results showed that $\mathrm{CoCl}_{2}$ inhibited mTOR phosphorylation in $\mathrm{H} 9 \mathrm{C} 2$ and $\mathrm{AC} 16$

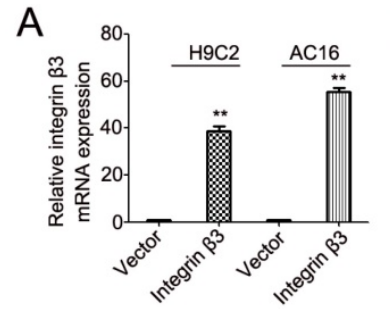

B
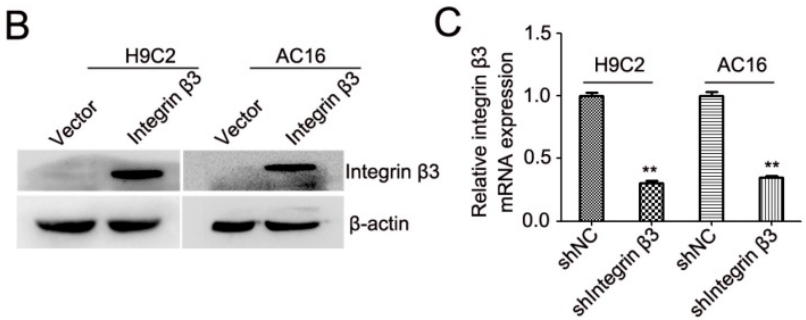

E
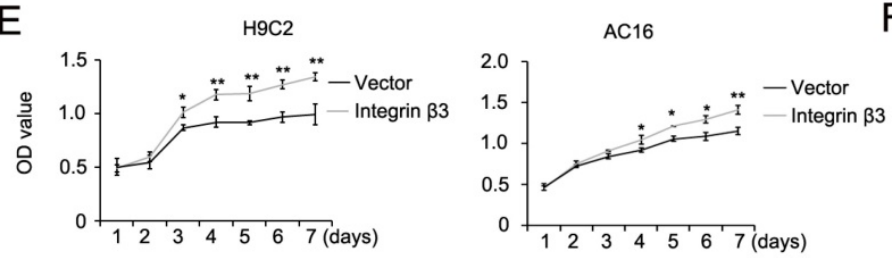

G
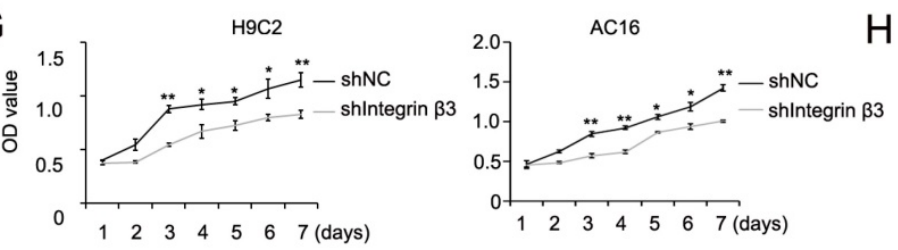

$\mathrm{F}$

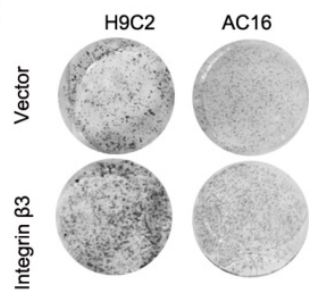

$\mathrm{H}$
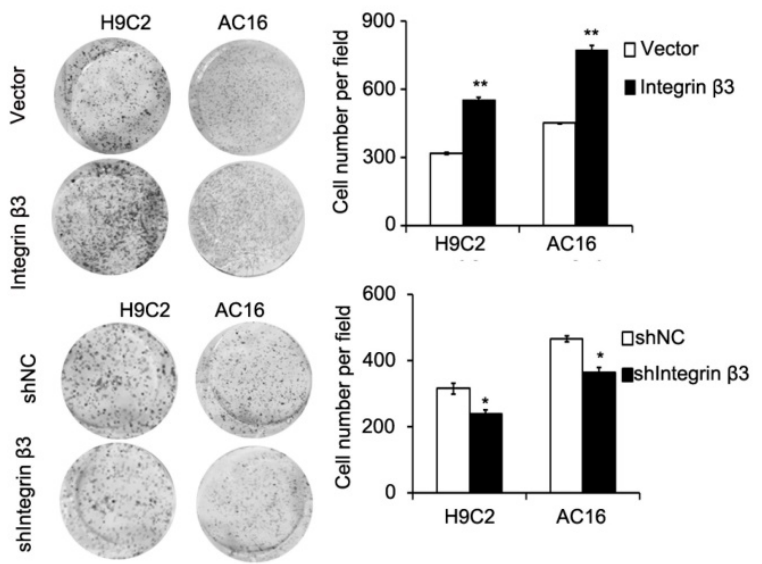
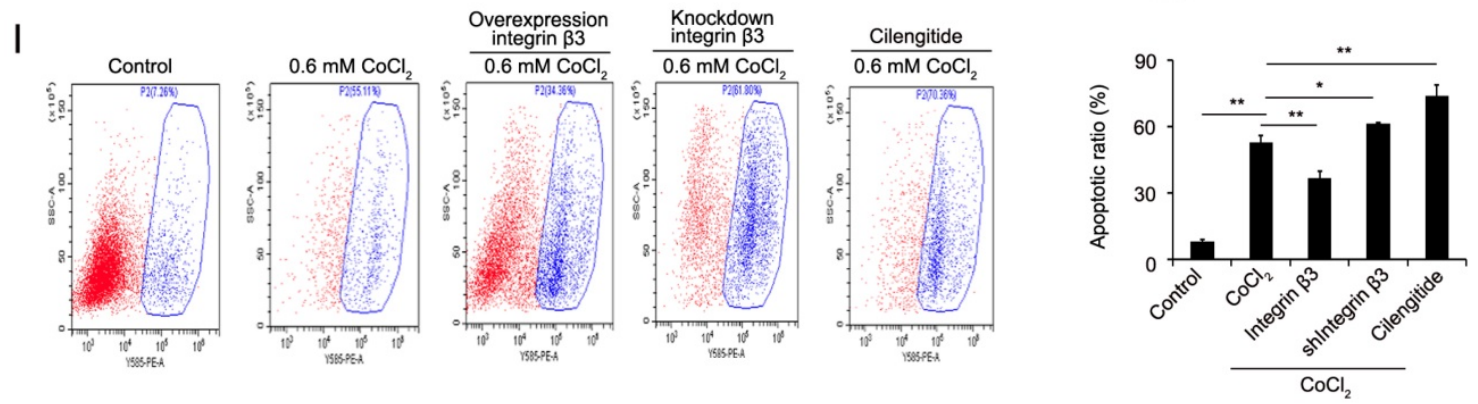

Figure 2. Integrin $\beta 3$ increases cardiomyocyte proliferation and weakens $\mathbf{C o C l}_{2}$-induced apoptosis. (A) The mRNA levels of integrin $\beta 3$ in $\mathrm{H} 9 \mathrm{C} 2$ and $A C 16$ cells expressing empty vector or integrin $\beta 3$ was detected by qRT-PCR. (B) The protein levels of integrin $\beta 3$ in $\mathrm{H} 9 \mathrm{C} 2$ and $A C 16$ cells expressing empty vector or integrin $\beta 3$ were detected by western blot. (C) The mRNA levels of Integrin $\beta 3$ in $\mathrm{H} 9 \mathrm{C} 2$ and AC1 6 cells expressing shNC vector or Integrin $\beta 3$ shRNA was detected by qRT-PCR. (B) The protein levels of integrin $\beta 3$ in $\mathrm{H} 9 \mathrm{C} 2$ and $\mathrm{AC} 16$ cells expressing shNC vector or integrin $\beta 3$ shRNA were detected by western blot. The overexpression of integrin $\beta 3$ increased cardiomyocyte proliferation (E) and colony-forming ability $(\mathrm{F})$ in $\mathrm{H} 9 \mathrm{C} 2$ and $\mathrm{AC} 16$ cells. The knockdown of integrin $\beta 3$ decreased cardiomyocyte proliferation (G) and colony-forming ability $(\mathrm{H})$ in $\mathrm{H} 9 \mathrm{C} 2$ and $\mathrm{AC} 16$ cells. (I) Integrin $\beta 3$-overexpressing, integrin $\beta 3$ knockdown, control and inhibitor cilengitide-treated (10 $\mu \mathrm{M}) \mathrm{H} 9 \mathrm{C} 2$ cells were treated with $\mathrm{CoCl}_{2}(0.6 \mathrm{mM})$ for $12 \mathrm{~h}$, and apoptosis was detected by flow cytometry. The bar graphs show the quantitative analysis data. $* P<0.05 ; * * P<0.05$. 
cells (Figure 5A). mTOR is activated by Akt which is associated with increased cell proliferation [14]. We next detected the expression of Akt phosphorylation in $\mathrm{H} 9 \mathrm{C} 2$ and AC16 cells. We found that phosphorylated Akt was decreased and PTEN expression was upregulated in $\mathrm{CoCl}_{2}$-treated cells (Figure 5A). In addition, we found that phosphorylated ERK1/2 was decreased in $\mathrm{CoCl}_{2}$-treated cardiomyocytes (Figure 5A, Figure S3). To confirm the role of Akt and ERK1/2 in
$\mathrm{CoCl}_{2}$-treated cardiomyocytes, the Akt inhibitor LY294002 and ERK1/2 inhibitor U0126 were used in $\mathrm{CoCl}_{2}$-treated cardiomyocytes. Our results showed that the inhibitory effects of $\mathrm{CoCl}_{2}$ on $\mathrm{H} 9 \mathrm{C} 2$ and $\mathrm{AC} 16$ cells were enhanced by LY294002 and U0126 (Figure 5B). Therefore, these results suggested that the hypoxia-induced inhibition of cardiomyocyte proliferation may be achieved through the PTEN/Akt/mTOR and ERK1/2 signaling pathways.

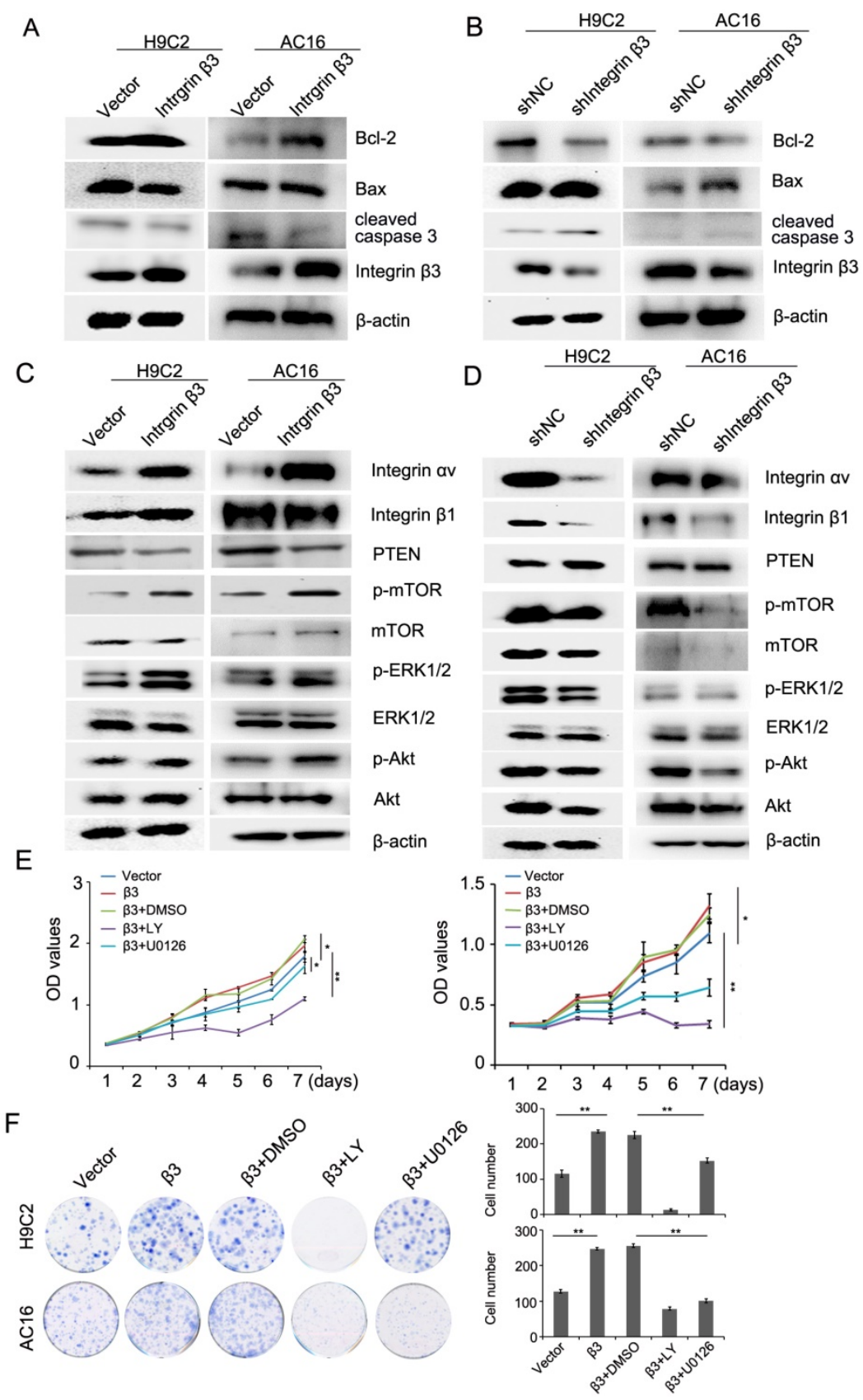

Figure 3. Integrin $\beta 3$ promotes cardiomyocyte proliferation through the regulation of the PTEN/Akt/mTOR and ERK1/2 pathways. (A) Bcl-2, Bax, integrin $\beta 3$ and cleaved caspase 3 expression was detected by western blotting in integrin $\beta 3$-overexpressing $\mathrm{H} 9 \mathrm{C} 2$ and $\mathrm{ACl} 16$ cells. (B) Bcl-2, Bax, integrin $\beta 3$ and cleaved caspase 3 expression was detected by western blotting in integrin $\beta 3$-knockdown $\mathrm{H} 9 \mathrm{C} 2$ and $A C 16$ cells. (C) Integrin $\beta 1$, integrin $\alpha v$, PTEN, p-Akt, Akt, p-ERK1/2, ERK1/2, p-mTOR and mTOR were detected by western blotting in integrin $\beta 3$-overexpressing $H 9 C 2$ and AC16 cells. (D) Integrin $\beta 1$, integrin $\alpha v, P T E N, p-A k t, A k t, p-E R K 1 / 2$, ERK1/2, $P$-mTOR and mTOR were detected by western blotting in integrin $\beta 3$-knockdown $\mathrm{H} 9 \mathrm{C} 2$ and AC16 cells. (E) Integrin $\beta 3$-overexpressing $\mathrm{H} 9 \mathrm{C} 2$ and $A C 16$ cells were treated with LY294002 $(10 \mu \mathrm{M})$ and $\mathrm{U} 0126(10 \mu \mathrm{M})$ for the indicated times. Cell proliferation was detected by the CCK8 assay. (F) Integrin $\beta 3$-overexpressing $\mathrm{H} 9 \mathrm{C} 2$ and $\mathrm{AC} 16 \mathrm{cells}$ were treated with LY294002 and U0126 for the indicated times. Cell proliferation was detected by the colony formation assay. $* P<0.05 ; * * P<0.01$. 
A
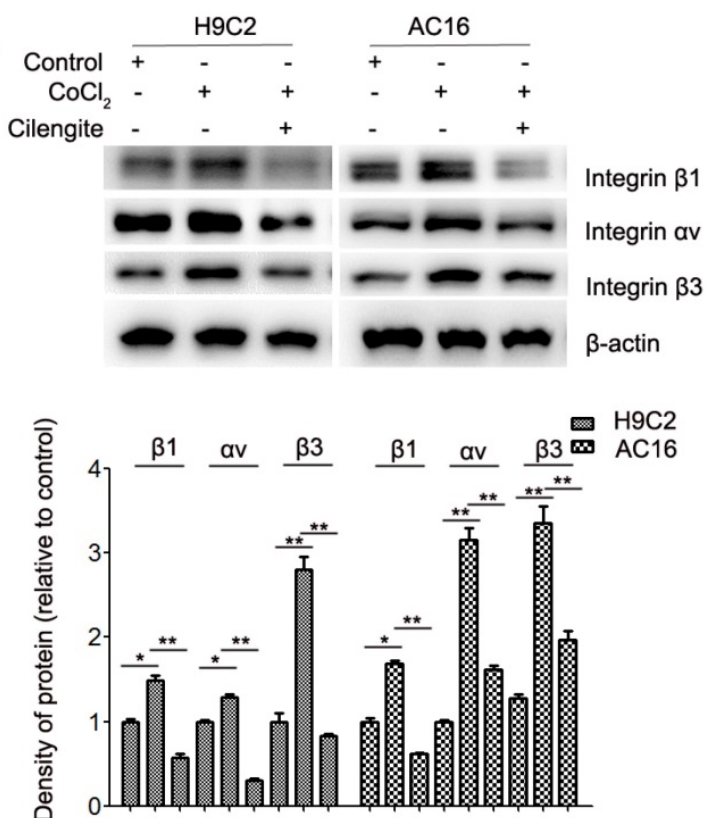

Control + - + + + - + - + - + -

$\mathrm{CoCl}_{2}+++-++-++-++-++-++$

Cilengite - - + + + + $++\cdots+\cdots+$

C

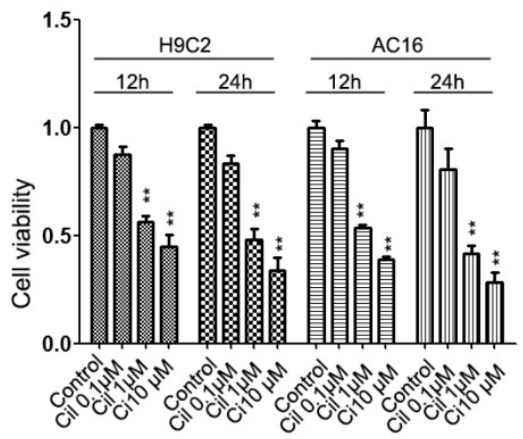

B
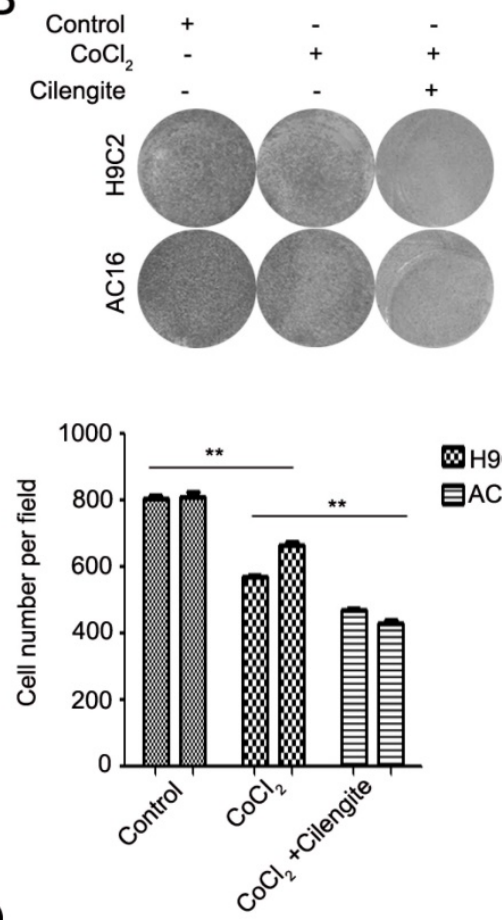

$\mathbf{m} 9 \mathrm{C} 2$ 目AC16

$\mathrm{D}$

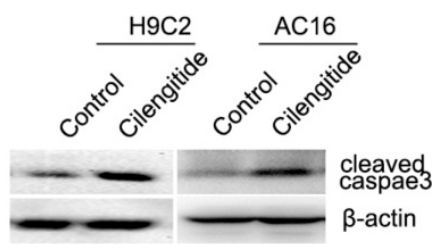

Figure 4. Cilengitide exacerbates the $\mathrm{CoCl}_{2}$-induced inhibition of cardiomyocytes. (A) The expression of integrin $\beta 3$, integrin $\beta 1$ and integrin $\alpha v$ was detected by western blotting in integrin $\beta 3$-overexpressing $\mathrm{H} 9 \mathrm{C} 2$ and $\mathrm{ACl} 16$ cells. (B) Cilengitide inhibited cardiomyocyte colony-forming ability in $\mathrm{CoCl}$-treated cells. (C) $\mathrm{H} 9 \mathrm{C} 2$ and $\mathrm{ACl}$ cells were treated with different dose of cilengitide. Cell viability was detected by the CCk8 assay. (D) The expression of cleaved caspase 3 in H9C2 and AC16 cells treated with cilengitide $(10 \mu \mathrm{M})$ for $24 \mathrm{~h} . * P<0.05 ; * * P<0.01$.

\section{Integrin $\beta 3$ promotes cardiomyocyte proliferation through the regulation of the PTEN/Akt/mTOR and ERK1/2 signaling pathways}

The above- mentioned results showed that hypoxia-induced cardiomyocyte apoptosis may be achieved through the PTEN/Akt/mTOR and ERK1/2 signaling pathways. Thus, we hypothesized that integrin $\beta 3$ may promote proliferation by regulating the PTEN/Akt/mTOR and ERK1/2 signaling pathways. Therefore, we next detected the levels of PTEN/Akt/mTOR and the ERK1/2 signaling pathway. Our results showed that the overexpression of integrin $\beta 3$ increased the expression of p-Akt, p-ERK1/2, and p-mTOR and inhibited the expression of PTEN (Figure 3C, Figure S2C). Conversely, the knockdown of integrin $\beta 3$ decreased the expression of p-Akt, p-ERK1/2, p-mTOR and increased expression of PTEN in H9C2 and AC16 cells (Figure 3D, Figure S2D). To confirm these results, we used the Akt inhibitor LY294002 and the ERK1/2 inhibitor U0126 in integrin $\beta 3$ overexpressing $\mathrm{H} 9 \mathrm{C} 2$ and $\mathrm{AC} 16$ cells. Our results showed that LY294002 and U0126 suppressed proliferation and clone-forming ability (Figure $3 \mathrm{E}$ and $3 \mathrm{~F}$ ). Therefore, these results indicated that integrin $\beta 3$ promotes cardiomyocyte proliferation through regulation of the PTEN/Akt/mTOR and ERK1/2 signaling pathways.

\section{Integrin $\alpha v$ and integrin $\beta 1$ coordinate with integrin $\beta 3$ to mediate cardiomyocyte proliferation}

A previous report showed that integrin $\beta 1$ and integrin $\beta 3$ can bind to integrin av to form heteromers [12]. We found that the expression of integrin $\beta 1$ and integrin av was upregulated in integrin $\beta 3$-overexpressing cardiomyocytes. The expression of 
integrin $\beta 1$ and integrin av was decreased in integrin $\beta 3$ knockdown cardiomyocytes. Therefore, we speculated whether integrin $\beta 1$ and integrin av play an important role in integrin $\beta 3$-induced cardiomyocyte proliferation. Furthermore, the colocalization of integrin $\beta 3$ and integrin av was found in cardiomyocytes using confocal microscopy (Figure 6A). Next, we used shRNA to decrease the expression of integrin av in integrin $\beta 3$ overexpressing cardiomyocytes (Figure 6B). The results showed that silencing integrin av weakened the effect of integrin $\beta 3$ on cardiomyocyte proliferation and clone-forming ability (Figure 6C and 6D). Furthermore, the expression of p-Akt, p-ERK1/2 and $\mathrm{p}$-mTOR was slightly inhibited in integrin av knockdown cardiomyocytes (Figure 6E).

To confirm the role of integrin $\beta 1$ in integrin $\beta 3$-mediated cardiomyocyte proliferation, the expression of integrin $\beta 1$ was inhibited using two integrin $\beta 1$ shRNAs (Figure 7A, Figure S4). Our results showed that knockdown of integrin $\beta 1$ weakened the effect of integrin $\beta 3$ on cardiomyocyte proliferation and clone-forming ability in $\mathrm{H} 9 \mathrm{C} 2$ and AC16 cells (Figure 7B and 7C). Meanwhile, the expression of p-Akt, p-ERK1/2 and p-mTOR was slightly inhibited in integrin $\beta 1$ knockdown cardiomyocytes (Figure 7A). Therefore, we speculated that integrin $\beta 1$ and integrin av coordinates with integrin $\beta 3$ to mediate cardiomyocyte proliferation.

\section{Discussion}

In the present study, we found that the expression of integrin $\beta 3$ was upregulated in hypoxia-induced cardiomyocyte apoptosis in vitro and in vivo. The overexpression of integrin $\beta 3$ inhibited $\mathrm{CoCl}_{2}$-induced cardiomyocyte apoptosis, whereas knocking down integrin $\beta 3$ expression using shRNA or the integrin $\beta 3$ inhibitor cilengitide exacerbates cobaltous chloride-induced cardiomyocyte apoptosis. Furthermore, we found that integrin $\beta 3$ promotes cardiomyocyte proliferation through the regulation of the PTEN/Akt/mTOR and ERK1/2 signaling pathways. In addition, we found that integrin $\beta 1$ coordinated with integrin $\beta 3$ to mediate cardiomyocytes proliferation. Thus, the results of the present study demonstrated the mechanism by which integrin $\beta 3$ protects against cardiomyocyte apoptosis.

Integrin $\beta 3$, a well-known member of the integrin family, has been studied extensively in cell proliferation and metastasis in various cancers [15, 16]. Recent studies have shown that integrin $\beta 3$ plays an important role in cardiovascular diseases. Liu et al reported that inhibiting integrin $\beta 3$ reduces the attachment, retention and therapeutic benefits of human cardiospheres in mice with acute myocardial infarction [8]. Misra et al. reported that enhanced integrin $\beta 3$ signaling is a crucial link between elastin deficiency and arterial hypermuscularization and that integrin $\beta 3$ blockade is a promising and much needed noninvasive therapeutic approach for supravalvular aortic stenosis [17]. Integrin $\beta 3$ knockout mice develop mild cardiac hypertrophy and inflammation [18]. In this study, we found that integrin $\beta 3$ was upregualted in AMI and CHF rat myocardial tissues. The results are consistent with previous reports $[9$, 19]. In addition, knocking down integrin $\beta 3$ also increases apoptosis in cardiomyocytes treated with hydrogen peroxide [20]. Therefore, these results suggested that integrin $\beta 3$ plays a protective role in cardiomyocyte apoptosis.
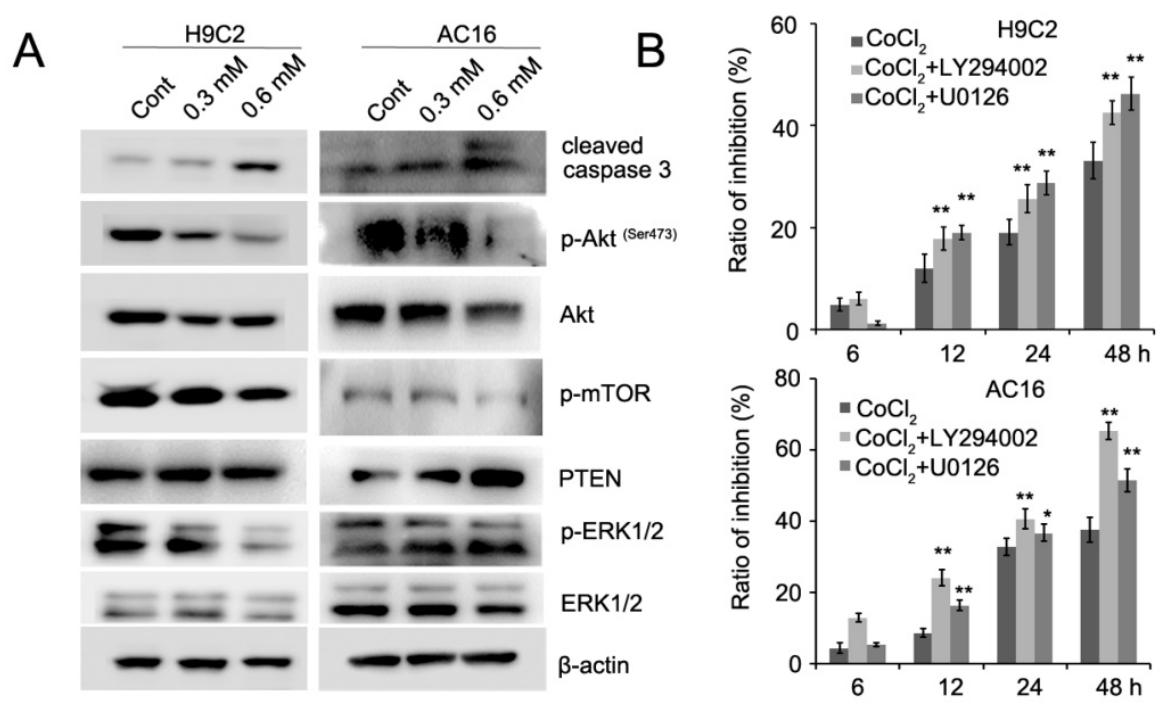

Figure 5. $\mathrm{CoCl}_{2}$ inhibits cardiomyocyte proliferation through the regulation of the PTEN/Akt/mTOR and ERK1/2 pathways. (A) p-Akt, Akt, $\mathrm{p}-\mathrm{ERK} 1 / 2$, ERK $1 / 2$, $\mathrm{P}$-mTOR, mTOR and cleaved caspase 3 were detected by western blotting in $\mathrm{CoCl}_{2}$-treated $\mathrm{H} 9 \mathrm{C} 2$ and $\mathrm{AC} 16$ cells. (B) $\mathrm{CoCl} 2$-treated $\mathrm{H} 9 \mathrm{C} 2$ and $\mathrm{ACl} 16$ cells were treated with LY294002 and U0126 for the indicated times. Cell proliferation was detected by the CCK8 assay. $* P<0.05 ; * * P<0.01$. 
A
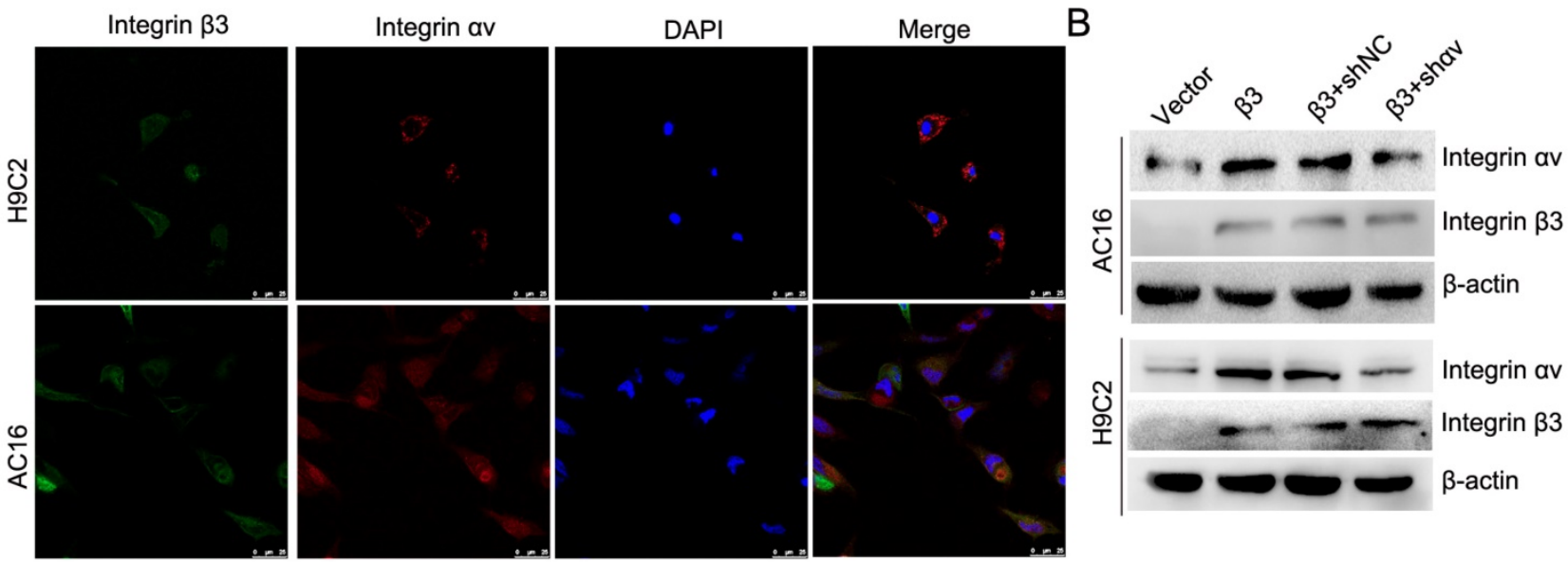

C
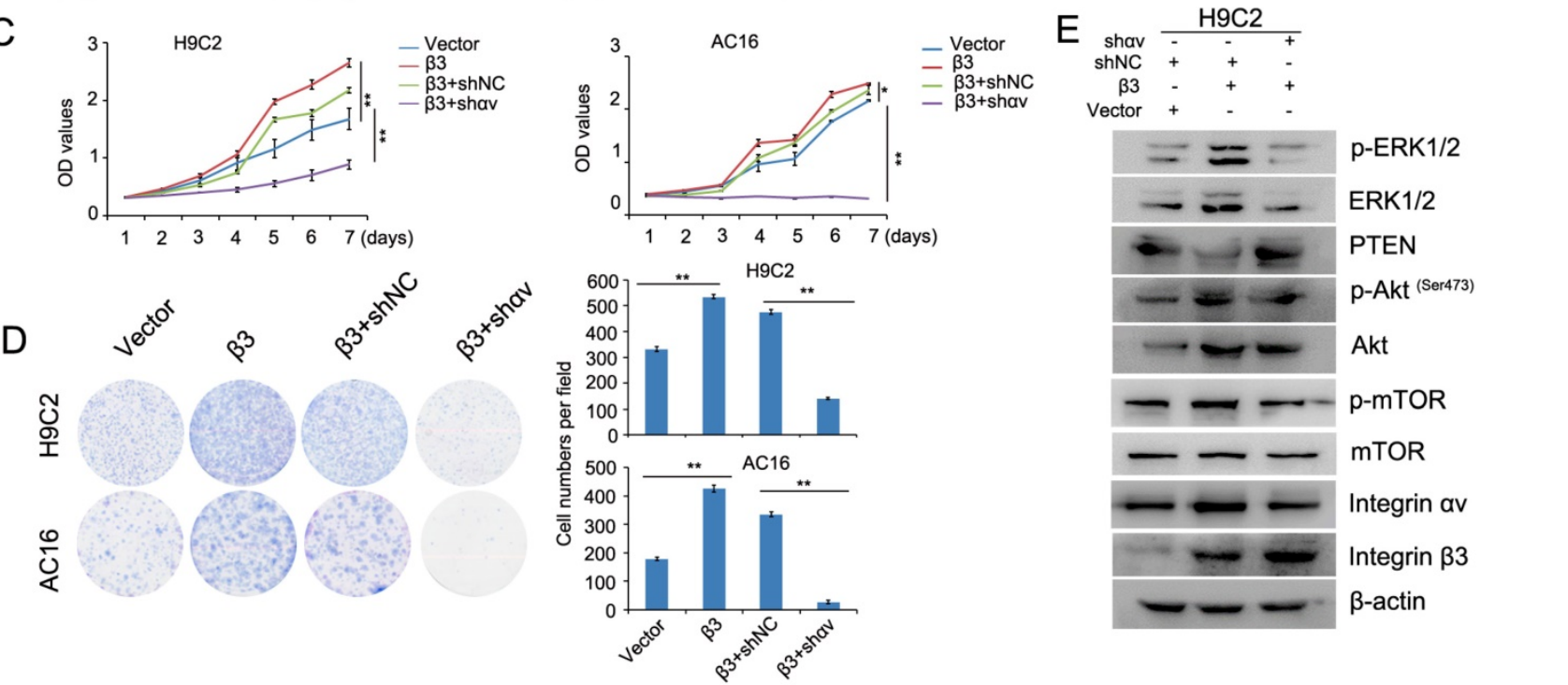

Figure 6. Knockdown of integrin $\alpha \mathrm{v}$ weakens the effect of integrin $\mathrm{B3}$ on cardiomyocyte proliferation and clone-forming ability in $\mathrm{H} 9 \mathrm{C} 2$ and $\mathrm{AC} 16 \mathrm{cells}$. (A) Confocal microscopy was used to detect the localization of integrin $\beta 3$ and integrin $\alpha v$ in $\mathrm{H} 9 \mathrm{C} 2$ and $\mathrm{ACl} 16$ cells. (B) Integrin $\beta 3$-overexpressing $\mathrm{H} 9 \mathrm{C} 2$ and $\mathrm{AC} 16$ cells were transfected with integrin $\alpha v$ shRNA as indicated, and the expression of integrin $\beta 1$ and integrin $\beta 3$ was detected by western blotting. Cell proliferation (C) and colony-forming ability (D) were measured by the CCK8 assay and colony formation assay. (E) Integrin $\beta 3$-overexpressing $\mathrm{H} 9 \mathrm{C} 2$ cells were transfected with integrin $\alpha \mathrm{v}$ shRNA as indicated, and the expression of integrin $\beta 3$, integrin $\alpha \mathrm{v}, \mathrm{mTOR}, \mathrm{p}$-mTOR, Akt, $\mathrm{p}-\mathrm{Akt}$, PTEN, ERK1/2 and $\mathrm{p}$-ERk1/2 were detected by western blotting. $* P<0.05$, $* * P<0.01$.

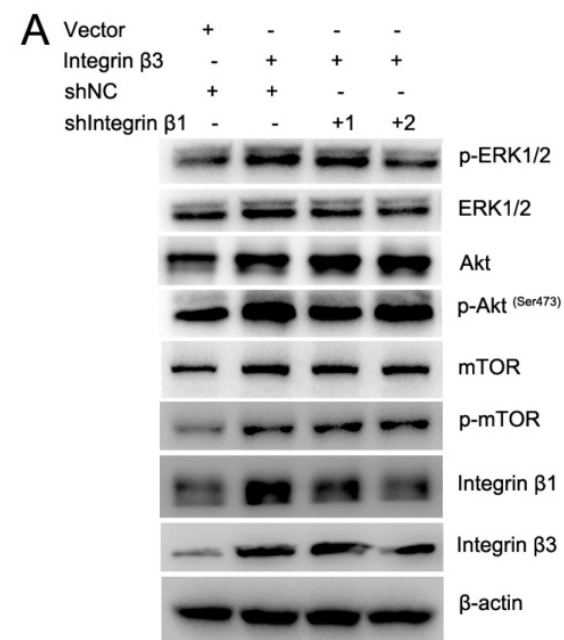

B
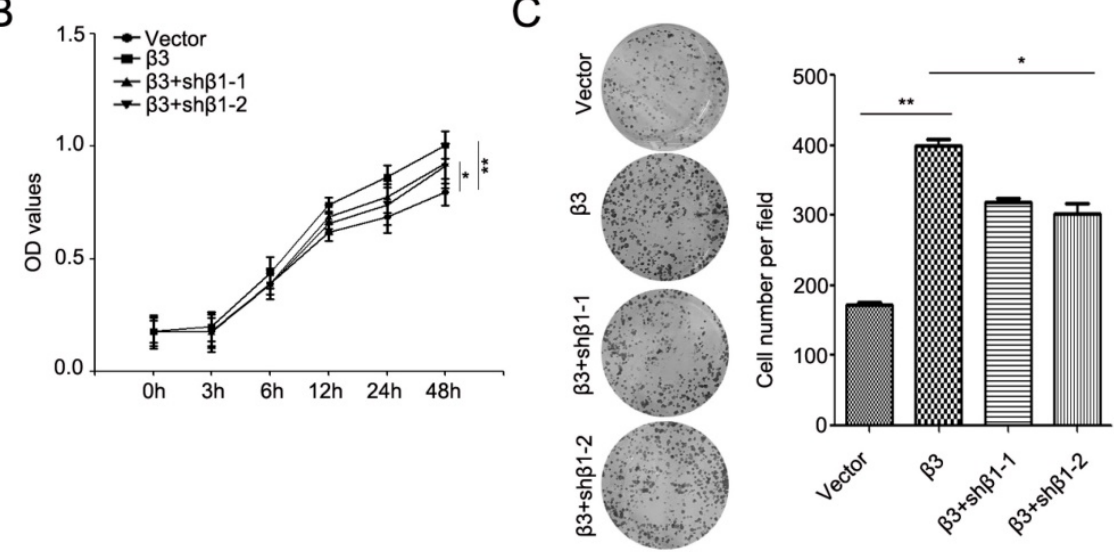

Figure 7. Knockdown of integrin $\beta 1$ weakens the effect of integrin $\beta 3$ on cardiomyocyte proliferation and clone-forming ability in $\mathrm{H} 9 \mathrm{C} 2$ cells. (A) Integrin $\beta 3$-overexpressing $\mathrm{H} 9 \mathrm{C} 2$ cells were transfected with integrin $\beta 1$ shRNA as indicated, and the expression of $p-A k t, A k t, p-E R K 1 / 2, E R K 1 / 2$, p-mTOR, mTOR, integrin $\beta 1$ and integrin $\beta 1$ were detected by western blotting. Cell proliferation $(B)$ and colony-forming ability $(C)$ was measured by the CCK 8 assay and colony formation assay. $* P<0.05$, $* * p<0.01$. 
Akt is known as a serine/threonine kinase that plays a vital role in the regulation of cell survival, proliferation, angiogenesis, and metabolism [21]. Our data demonstrated that the expression of integrin $\beta 3$ is upregulated in $\mathrm{CoCl}_{2}$-treated cardiomyocytes and that this upregulation is accompanied by the downregulation of phosphorylated Akt. Furthermore, we found that the overexpression of integrin $\beta 3$ increased phosphorylated Akt, whereas knockdown of integrin $\beta 3$ decreased phosphorylated Akt in H9C2 and AC16 cells. Therefore, integrin $\beta 3$ promotes cardiomyocyte proliferation through regulation of Akt phosphorylation. Following activation, Akt directly activates mammalian target of rapamycin complex 1 (mTORC1) by phosphorylating mTORC1 at Ser2448 [22]. In addition, PTEN negatively controls Akt activation, resulting in decreased recruitment of Akt to the cell membrane [21]. Our results also showed that the overexpression of integrin $\beta 3$ activated phosphorylated mTOR and inhibited PTEN expression. Furthermore, similar results were also observed in AMI and CHF rat models. Johnston et al. used integrin $\beta 3$ knockout mice to demonstrate that integrin $\beta 3$ is critical for activating NF-kB-mediated cell survival signaling in cardiomyocytes [23]. NF-KB is a downstream component of the PI3K/Akt pathway, and is activated by PI3K/Akt pathway through the phosphorylation of IKB kinase (IKK), leading to IKB degradation [24]. Therefore, we consider that integrin $\beta 3$ increases cardiomyocyte proliferation through the regulation of PTEN/Akt/mTOR pathway, leading to NF-kB activation.

The ERK1/2 signaling pathway also plays animportant role in the regulation of cell survival and proliferation. We also found that the overexpression of integrin $\beta 3$ increases ERK1/2 phosphorylation in cardiomyocytes. Previous studies have shown that crosstalk may occur between the PI3K and ERK1/2 pathways $[25,26]$. Therefore, we speculate that integrin $\beta 3$ promotes cardiomyocyte proliferation through the regulation of PTEN/Akt/mTOR and ERK1/2 signaling pathways.

Precious studies have shown that integrin $\beta 1$ and integrin $\beta 3$ can bind to integrin av to form heteromers [12]. In this study, we found that the overexpression of integrin $\beta 3$ increases integrin $\beta 1$ expression, whereas the knockdown of integrin $\beta 3$ inhibited integrin $\beta 1$ expression. To exclude the role of integrin $\beta 1$ in integrin $\beta 3$-induced cell proliferation, integrin $\beta 1$ shRNA was used during integrin $\beta 3$-induced cell proliferation. We found that knockdown of integrin $\beta 1$ weakened the effect of integrin $\beta 3$ on cardiomyocyte proliferation and clone-forming ability in $\mathrm{H} 9 \mathrm{C} 2$ and AC16 cells. Therefore, we believe that integrin $\beta 1$ coordinates with integrin $\beta 3$ to mediate cardiomyocyte proliferation.

\section{Supplementary Material}

Supplementary figures.

http://www.ijbs.com/v16p0644s1.pdf

\section{Acknowledgements}

This work was supported by the National Natural Science Foundation (81770505, 81972581, 81472570), Research Project of Shanghai Municipal Health and Family Planning Commission (201740060).

\section{Competing Interests}

The authors have declared that no competing interest exists.

\section{References}

1. Rochette L, Malka G, Cottin Y. Hypoxia and heart regeneration: A new paradoxical approach for cardioprotection. Arch Cardiovasc Dis 2017, 110: 503-507.

2. Cooper J, Giancotti FG. Integrin Signaling in Cancer: Mechanotransduction, Stemness, Epithelial Plasticity, and Therapeutic Resistance. Cancer Cell 2019, 35: 347-367.

3. Moreno-Layseca P, Icha J, Hamidi H, et al. Integrin trafficking in cells and tissues. Nat Cell Biol 2019, 21: 122-132.

4. Harston RK, Kuppuswamy D. Integrins are the necessary links to hypertrophic growth in cardiomyocytes. J Signal Transduct 2011, 2011: 521742.

5. Noh KW, Sohn I, Song JY, et al. Integrin beta3 Inhibition Enhances the Antitumor Activity of ALK Inhibitor in ALK-Rearranged NSCLC. Clin Cancer Res 2018, 24: 4162-4174.

6. Suryakumar G, Kasiganesan H, Balasubramanian S, et al. Lack of beta3 integrin signaling contributes to calpain-mediated myocardial cell loss in pressure-overloaded myocardium. J Cardiovasc Pharmacol 2010, 55: 567-573.

7. Zhu Y, Li L, Gong S, et al. $\beta 3$-integrin inhibits lipopolysaccharide-induced autophagy in cardiomyocytes via the Akt signaling pathway. Cardiology 2015, 130: 249-259.

8. Liu S, Jiang Z, Qiao L, et al. Integrin beta-3 is required for the attachment, retention and therapeutic benefits of human cardiospheres in myocardial infarction. J Cell Mol Med 2018, 22: 382-389.

9. Su Y, Tian H, Wei L, et al. Integrin beta3 inhibits hypoxia-induced apoptosis in cardiomyocytes. Acta Biochim Biophys Sin (Shanghai) 2018, 50: 658-665.

10. Jiang Z, Zhou $Q, G e C$, et al. Rpn10 promotes tumor progression by regulating hypoxia-inducible factor 1 alpha through the PTEN/Akt signaling pathway in hepatocellular carcinoma. Cancer Lett 2019, 447: 1-11.

11. Tian H, Ge C, Zhao F, et al. Downregulation of AZGP1 by Ikaros and histone deacetylase promotes tumor progression through the PTEN/Akt and CD44s pathways in hepatocellular carcinoma. Carcinogenesis 2017, 38: 207-217.

12. Cai $W$, Chen $X$. Anti-angiogenic cancer therapy based on integrin alphavbeta3 antagonism. Anticancer Agents Med Chem 2006, 6: 407-428.

13. Martelli AM, Buontempo F, McCubrey JA. Drug discovery targeting the mTOR pathway. Clin Sci (Lond) 2018, 132: 543-568.

14. Janku F, Yap TA, Meric-Bernstam F. Targeting the PI3K pathway in cancer: are we making headway? Nat Rev Clin Oncol 2018, 15: 273-291.

15. Wen S, Hou Y, Fu L, et al. Cancer-associated fibroblast (CAF)-derived IL32 promotes breast cancer cell invasion and metastasis via integrin beta3-p38 MAPK signalling. Cancer Lett 2019, 442: 320-332.

16. Parvani JG, Gujrati MD, Mack MA, et al. Silencing beta3 Integrin by Targeted ECO/siRNA Nanoparticles Inhibits EMT and Metastasis of Triple-Negative Breast Cancer. Cancer Res 2015, 75: 2316-2325.

17. Misra A, Sheikh AQ, Kumar A, et al. Integrin beta 3 inhibition is a therapeutic strategy for supravalvular aortic stenosis. J Exp Med 2016, 213: 451-463.

18. Ren J, Avery J, Zhao H, et al. Beta3 integrin deficiency promotes cardiac hypertrophy and inflammation. J Mol Cell Cardiol 2007, 42: 367-377.

19. Sun M, Opavsky MA, Stewart DJ, et al. Temporal response and localization of integrins beta1 and beta 3 in the heart after myocardial infarction: regulation by cytokines. Circulation 2003, 107: 1046-1052.

20. Shewchuk LJ, Bryan S, Ulanova M, et al. Integrin beta3 prevents apoptosis of HL-1 cardiomyocytes under conditions of oxidative stress. Can J Physiol Pharmacol 2010, 88: 324-330.

21. Noorolyai S, Shajari N, Baghbani E, et al. The relation between PI3K/AKT signalling pathway and cancer. Gene 2019, 698: 120-128.

22. Hennessy BT, Smith DL, Ram PT, et al. Exploiting the PI3K/AKT pathway for cancer drug discovery. Nat Rev Drug Discov 2005, 4: 988-1004. 
23. Johnston RK, Balasubramanian S, Kasiganesan $\mathrm{H}$, et al. Beta3 integrin-mediated ubiquitination activates survival signaling during myocardial hypertrophy. FASEB J 2009, 23: 2759-2771.

24. Yang $\mathrm{L}, \mathrm{Hu} \mathrm{X}, \mathrm{Mo} \mathrm{YY}$. Acidosis promotes tumorigenesis by activating AKT/NF-kappaB signaling. Cancer Metastasis Rev 2019, 38:179-188

25. Aksamitiene E, Kiyatkin A, Kholodenko BN. Cross-talk between mitogenic Ras/MAPK and survival PI3K/Akt pathways: a fine balance. Biochem Soc Trans 2012, 40: 139-146.

26. Dent P. Crosstalk between ERK, AKT, and cell survival. Cancer Biol Ther 2014, 15: $245-246$ 